\title{
Expanded norms for 400 experimental pictures in an Argentinean Spanish-speaking population
}

\author{
Laura Manoiloff, Marcela Artstein, María Belén Canavoso, and Laura Fernández \\ Universidad Nacional de Córdoba, Córdoba, Argentina \\ AND \\ JUAN SEgui \\ CNRS and Université René Descartes, Paris, France
}

\begin{abstract}
The study of the cognitive processes in the production of language demands careful selection of stimuli and requires normative databases. The main goal of the present research was to collect normative data for the set of 400 figures taken from Cycowicz, Friedman, Rothstein, and Snodgrass (1997; including the 260 figures of Snodgrass \& Vanderwart, 1980) using a sample of native Argentinean Spanish speakers. The pictures have been standardized on the following variables: name agreement, image agreement, familiarity, visual complexity, image variability, age of acquisition, and word association. The obtained norms were compared with the normative data of other studies in Spanish, English, and French. This comparison highlights the variability of some of the measures (e.g., name agreement in naming and verbal association) across the different studies and confirms the necessity of elaborating specific norms that are adapted to the studied population's linguistic and sociocultural context. The norms described may be downloaded as supplemental materials for this article from http://brm.psychonomic-journals.org/content/supplemental.
\end{abstract}

In the field of psycholinguistics, one of the most used paradigms in the study of the production of isolated words has been picture naming. This experimental task is affected by different factors that influence the various stages of the picture-naming process. Researchers have tried to characterize and quantify these factors by means of specific variables that have proven to be good predictors of the speed and accuracy with which the task of picture naming is performed (Alario et al., 2004; Barry, Morrison, \& Ellis, 1997; Bonin, Boyer, Méot, Fayol, \& Droit, 2004; Cuetos, Ellis, \& Alvarez, 1999; Iyer, Saccuman, Bates, \& Wulfeck, 2001).

The establishment of norms related to these variables is an essential stage prior to the study of picture naming in a particular population. For example, diverse studies have been carried out with Snodgrass and Vanderwart's (1980) set of pictures with Spanish speakers from Spain (Cuetos et al., 1999; Sanfeliu \& Fernández, 1996), Mexico (Aveleyra, Gómez, Ostrosky, \& Rigalt, 1996; Bates et al., 2003), and Cuba (Manzano, Piñeiro, \& Reigosa, 1997). Although Spanish speakers share a basic corpus of lexical and grammatical knowledge, they show some global and particular discrepancies among them (Pérez \& Navalón, 2003).

Furthermore, the studies carried out in the Spanish language have worked with only a few variables (mainly, name agreement, image variability, familiarity, and visual complexity) and with a relatively small number of images (in a range from 140 to 260 images). Moreover, some of them used a small sample of participants to obtain the norms of different psycholinguistics variables. This is described more precisely in subsequent paragraphs.

For this reason, a normative study for more pictorial stimuli (400) was conducted in our laboratory with an Argentinean sample of participants, to establish the values corresponding to seven important variables in picture naming: visual complexity, image agreement, image variability, familiarity, word association, name agreement, and age of acquisition.

First, we will define these variables and discuss some empirical and theoretical information.

\section{Visual Complexity (Comp.)}

This variable refers to the number of lines and details in the drawing. Pictures with a higher visual complexity value are responded to more slowly than are those with a lower visual complexity value (Ellis \& Morrison, 1998). It has been suggested that this factor essentially affects the early stages of the visual processing of the picture (Alario \& Ferrand, 1999).

\section{Image Agreement (IA)}

This variable refers to the agreement or degree of concordance between the mental image of the object and its 
corresponding pictorial representation. Barry et al. (1997) showed that pictures with higher ratings of image agreement are processed faster than those with lower ratings. According to Alario and Ferrand (1999), this factor has its influence at the level of object recognition.

\section{Image Variability (IV)}

This variable refers to the average number of different pictures evoked by the name of an object. Alario et al. (2004) noted that pictures corresponding to objects whose names arouse higher IV values are processed faster than those that arouse lower IV values. These authors suggested that IV characterizes the richness of the semantic representation of object pictures.

\section{Familiarity (FAM)}

This variable refers to the acquaintance with the concept represented by the picture and is defined as the degree to which the participant comes in contact with, or thinks about, the thing depicted in daily life. Some studies (Cuetos et al., 1999; Ellis \& Morrison, 1998; Snodgrass \& Yuditsky, 1996) have reported that familiarity ratings are important predictors of picture-naming time, so that the concepts of objects considered more familiar are named faster than those considered less familiar. It has been suggested that the concept of familiarity affects the simplicity with which the representation of an object can activate its central semantic representation (Hirsh \& Funnell, 1995).

\section{Word Association (WA)}

This variable refers to the frequency with which a word is freely associated with another word, where the name of an image is the stimulus word. According to Fernández, Diez, Alonso, and Beato (2004), manipulation of the associative power between two words, using values provided by WA, has proven to have a reliable effect of semantic facilitation in priming studies and in memory recall tasks. Those authors proposed that the importance of determining WA is derived from the fact that these values are indicators of a strong connection between the representations of two words in the semantic memory. Barbón and Cuetos (2006), however, proposed that WA might be measuring access to the lexicon and not a connection at the semantic level, since this task could be based mainly on the spontaneous occurrence of words in daily life.

\section{Name Agreement (NA)}

This variable indicates the degree to which participants agree on the name of the picture. Name agreement is measured by the number of names given to a particular picture across participants. A picture that elicits many different names is considered to have a low NA, and one that elicits a single name is considered to have a high NA. Numerous studies (Alario et al., 2004; Barry et al., 1997; Bonin et al., 2004; Cuetos et al., 1999; Dell'Acqua, Lotto, \& Job, 2000; Ellis \& Morrison, 1998; Snodgrass \& Yuditsky, 1996; Vitkovitch \& Tyrrell, 1995) reported that this factor is a powerful predictor of naming speed, since, if a picture elicits many plausible names, the naming is slowed by the need to choose one from among the alternative names.
Pictures with only one predominating response, instead, are named faster and more correctly than are those with multiple responses. It has been suggested that this variable affects the retrieval stage of the phonological form of the name (Alario \& Ferrand, 1999).

\section{Age of Acquisition (AoA)}

This variable is defined as the age at which a word is learned, either orally or in writing. The data provided by AoA can be objective or subjective. The former determines the "real" age at which different words were acquired. The latter considers an estimation made by adults about when the words were acquired. In the study conducted by Iyer et al. (2001), it is stated that the results collected by subjective methods can reliably replace those collected by objective methods.

When the usefulness of AoA as a predictor of naming speed is evaluated, important controversies are found when determining the influence of AoA in comparison with that of the frequency of word use. It has been found that both factors are strongly associated; that is, if a word (corresponding to the name of an object) was acquired at an early age and that word has a high frequency, the naming time of the picture is shorter than it is in cases in which the word was acquired later and has a low frequency of use. Several studies have tried to determine whether one variable is responsible for the effects attributed to the other. For example, Ellis and Morrison (1998), Morrison, Chappell, and Ellis (1997), and Morrison, Ellis, and Quinlan (1992) have suggested that AoA is more important than frequency. However, more recent investigations have found that each variable makes an independent contribution to naming times (Alario et al., 2004; Barry et al., 1997; Morrison \& Ellis, 1995; Snodgrass \& Yuditsky, 1996).

As regards the stage in the process in which AoA exerts its influence, Barbón and Cuetos (2006) proposed that the effects are situated in the access to the lexicon and not in the semantic system - that is, in the activation of the phonological form corresponding to each meaning. However, more recent work by Dent, Johnston, and Humphreys (2008) has suggested that the AoA would affect earlier stages in the picture-naming process, such as the level of object recognition, semantic activation, and lemma selection.

Considering what has been outlined above, these variables can be considered to be predictors of the different stages in the picture-naming process. Moreover, each stage of the process is affected by more than one variable. Comp. and IA affect the retrieval stage of structural representations, since they stress the visual-perceptive aspect of the naming process. IV, WA, and FAM are considered to be variables that make it possible to know how the participant conceptualizes the picture to be named, and therefore they constitute the semantic aspect of the process. Finally, NA and AoA influence the retrieval time of the phonological representation of the name - that is, how the picture under study is named.

The strong influence of these variables on the picturenaming process makes it important to know the values of each. For this reason, many studies have been conducted 
on the basis of Snodgrass and Vanderwart's (1980) original set of pictures (or henceforth original set) in different languages and cultures (in British and American English, German, Portuguese, and in several Spanish-speaking countries, such as Spain, Mexico, and Cuba).

The information collected from the normative studies depends on the culture and language of the samples used. Sanfeliu and Fernández (1996) emphasized the need to extend Snodgrass and Vanderwart's (1980) original work on picture standardization to other contexts, because of the difficulty with generalization. They stated that objects that are common in a given culture might be less common in another culture and that pictures that evoke a very specific and concrete name in one language might evoke a more general term in another. Therefore, normative data must be established to fit with the language and the sociocultural context of the study's participants.

Antecedents of adaptations to Spanish of Snodgrass and Vanderwart's (1980) set of pictures are scarce. Among them is the one by Sanfeliu and Fernández (1996) in Spain, which used 254 pictures from the original set and evaluated the variables of NA, IA, FAM, IV, and Comp. on 261 students. Cuetos et al. (1999) took from the original set only 140 pictures and studied the naming time for those pictures according to the following variables: FAM, AoA, Comp., NA, IA, word length, and five frequency levels. Aveleyra et al. (1996) adapted the Snodgrass and Vanderwart set and worked with 88 Mexican students, who were divided into four groups for each variable to study: NA, IA, FAM, and Comp. Manzano et al. (1997) collected the responses to the set of pictures from Cuban participants in IA, FAM, Comp., and familiarity of the name. Bates et al. (2003) collected picture-naming norms (including latency, name agreement, visual complexity, goodness of depiction, frequency, word length, phonetic structure, and word complexity) for 520 black-and-white drawings (174 from the set proposed by Snodgrass \& Vanderwart, 1980) of common objects in seven different languages, including Spanish. In the last case, they obtained the data from a Mexican sample (50 students from Tijuana). However, to collect the data in the naming task, they used a different method than did other normative studies in Spanish, making it difficult to compare them. In their work, participants were instructed to speak the names of the pictures as quickly as possible. The target name (dominant response) for each picture was determined as valid or invalid according to the type of response (content) and the reaction time (RT) measure (usable; for more details, see Székely et al., 2003).

Regarding WA, only one study in Spanish (Fernández et al., 2004) has focused on this variable for the names of Snodgrass and Vanderwart's (1980) original set. The oral material used as stimuli in the association task consisted of the names given more frequently, according to Sanfeliu and Fernández (1996), who used the Snodgrass and Vanderwart set.

From the original set of Snodgrass and Vanderwart (1980), other studies have been published in which this set of pictures has been broadened to 400 pictures, such as the study by Cycowicz et al. (1997). The new pictures are more complex and are less common than those proposed by Snodgrass and Vanderwart; therefore, the expanded set of 400 pictures is considered to be more heterogeneous than the original one.

Alario and Ferrand (1999) adapted this set of 400 pictures for a French population, closely following the procedure proposed by Snodgrass and Vanderwart (1980) for this purpose and broadening the number of variables under study (NA, IA, FAM, Comp., IV, and AoA), thereby adding more information than the original work.

\section{The Present Study}

The main goal of the present study was to obtain a normative database for the 400 pictures taken from Cycowicz et al. (1997, which includes Snodgrass \& Vanderwart's [1980] set of 260 pictures) to have more pictures with more heterogeneous characteristics.

We included a larger number of variables than those considered in the previous works, in order to add information for picture selection and usage, especially in relation to WA, which is extremely important for priming studies. The data was collected from a local sample of adult Argentinean Spanish-speaking participants to control for the effects of language and culture in the usage of these pictures for future research.

Besides the collection of ratings for each of the pictorial stimuli in relation to the variables (name agreement, image agreement, familiarity, visual complexity, image variability, age of acquisition, and word association), we performed a descriptive analysis (measuring central tendency, variability, and dispersion) for each variable. Furthermore, we examined the existing relations among the variables predicting the production process and counted the proportion of errors made by the participants in relation to the different items in each variable, to determine which are the more problematic items and the variables that demand more effort from the participant to respond. Lastly, the results collected in this study were compared with those reported by previous investigations, not only as an indicator of validity as regards to the measures obtained and the procedure, but also to determine which variables are most affected by language and culture (Alario \& Ferrand, 1999; Dell'Acqua et al., 2000; Sanfeliu \& Fernández, 1996).

\section{METHOD}

\section{Participants}

A random sample of 253 psychology students ( $70 \%$ female, $30 \%$ male) from the National University of Cordoba, Argentina participated in this study. All were native Argentinean Spanish speakers. The sample was subdivided into seven groups with 35 participants (ranging in age from 18 to 26 years) for each task.

\section{Materials}

The set of 400 black-and-white line pictures was taken from Cycowicz et al. (1997). Each picture was printed on an individual slide.

\section{Design and Procedure}

Each variable (NA, IA, FAM, Comp., IV, AoA, and WA) was treated as an interparticipant factor - that is, each was evaluated by a group of participants, and, therefore, participants who answered to 
one variable did not participate in the evaluation of the others. Each variable was applied to a previously formed group.

The general procedure used was similar to Snodgrass and Vanderwart's (1980) and Alario and Ferrand's (1999) original, both in terms of the tasks performed and the way in which this was done. As in previous studies, pictures were projected sequentially, using an overhead projector on a large white screen at the front of a slightly darkened room, to a group of participants seated in a classroom. For each task, at the beginning of every session, the instructions were given to the participants in both written and oral form and they were encouraged to answer carefully and consistently. They were given individual answer sheets and were instructed to respond to every drawing. Thus, seven forms of the answer sheet were designed to register the responses, each of which contained the corresponding instructions for each test, a clarifying example, and the space where the participants had to write down or classify their response.

Nevertheless, Alario and Ferrand's (1999) study differed in the number of sessions that were used to evaluate the 400 images. Due to the high number of items in the present study, the test was divided into two sessions, which might increase the number of errors or the quantity of incomplete responses in the last pictures (Snodgrass \& Yuditsky, 1996). Consequently, we created two experimental lists of 200 figures. The figures were distributed between these lists in order to avoid presenting items that were evaluated in a similar way in the same session, according to previously published norms. In particular, an equilibrated distribution of the figures between the two lists was adopted concerning the name agreement factor, given that this factor constitutes a main determinant of accuracy and RT. This distribution was used to produce a semirandom order of presentation that was the same for all participants.

To obtain the values for WA, we followed the same steps described in the study by Fernández et al. (2004). We used the standard word association, according to which a word is presented to the participant, who is asked to respond with the first word that comes to mind. Although study words were presented in a written form in Fernández et al., in the present study, the words were read aloud.

For the NA, IA, FAM, and Comp. variables, our procedure was similar to the general procedure: Participants were told to evaluate each projected picture according to the corresponding instructions.

In the NA task, the participants were instructed to identify the drawing by writing the first word that came to mind on the answer sheet, on the dotted line following the number that corresponded to each object ("You will see different objects, and your task consists of writing down the name you think that corresponds to that object.")..$^{1}$ In addition, the corresponding examples in which filler stimuli and responses were used were given to clarify all doubts about their tasks. Each slide was presented for $7 \mathrm{sec}$. If participants could not name the object, they had to indicate the reason: "don't know the object" (DKO), "don't know the name" (DKN), or "tip of the tongue" (TOT, i.e., answer momentarily inaccessible; Alario \& Ferrand, 1999; Snodgrass \& Vanderwart, 1980). The information statistic, $H$, was computed using the formula defined by Snodgrass and Vanderwart (1980),

$$
H=\sum_{i=1}^{k} p_{i} \log _{2}\left(1 / p_{i}\right)
$$

where $k$ refers to the number of names given to the picture and $p_{i}$ indicates the proportion of participants who gave the name.

In the IA task, participants were asked to judge how closely each picture resembled their mental image of the object ("You will listen to the name of an object. Try to retain the mental image that is formed from the name heard. After a few seconds, a drawing of the object named will be projected, in order to compare how close or how far your mental image is from the projected drawing - that is, assess the degree of concordance between the two."). Before presenting each picture, the experimenter called out the picture's most common name (obtained from the NA task, which was completed before the IA task began), waited for $4 \mathrm{sec}$, and then projected the picture on the screen. The picture stayed on the screen for $3 \mathrm{sec}$, and participants rated the degree of agreement between their image and the picture using a 5-point scale, where $1=$ very low concordance and $5=$ very high concordance. If they could form no image, they were instructed to write the letters "NI" (no image); they were instructed to write the letters "DO" (different object) if they formed a mental image that corresponded to a different object.

In the FAM task, the familiarity was defined as the degree to which the participant came in contact with, or thought about, the thing depicted in daily life. They were told to evaluate the concept and not the picture ("You will see pictures of objects, and the task consists of determining how familiar the object is for you, according to how usual or unusual the object is in your realm of experience. Familiarity means the frequency with which you come into contact with the object or the frequency with which you think of it in your daily life."). Each picture was presented for $4 \mathrm{sec}$, and the participants were asked to judge familiarity on a 5-point scale, where $1=$ very strange object and $5=$ very familiar object. They were told to write "DKO" if they could not recognize the object.

For the Comp. task, participants were told to evaluate the composition of the picture (not the concept for which it stood); therefore, all the pictures could be evaluated independently of whether the object was known. The participants were instructed "You will see a picture. You should observe carefully, because your task will be to rate the complexity of the design of the picture, rather than the complexity of the object it represents. The complexity is defined as the number of details or lines that compose a picture." The pictures stayed on the screen for $4 \mathrm{sec}$, with a 3-sec interval between them. They evaluated each picture according to a 5 -point scale, where $1=$ very simple and $5=$ very complex.

For the remaining variables (IV, AoA, and WA), the names were presented written or orally, according to the task, and the participants were told to evaluate them following the corresponding instruction.

In the IV task, participants evaluated the image variability potential of the name. For this task, they had to estimate the number of different images they imagined from the given name ("You will listen to the name of an object and try to determine the number of mental images that come to mind. The variability refers to the number of pictures evoked by the name of an object. The greater the number of images evoked indicates greater variability."). Participants responded using a $1-5$ point scale, where $1=$ very low variability and $5=$ very high variability. Participants were told to write "DKO" if they did not know the object by the name given.

In the AoA task, participants performed a subjective estimate of the age at which they had learned each of the names, in its written or oral form. For this, they were given a list with the names and a 5 -point scale, where $1=0-3$ years old, $2=3-6$ years old, $3=$ 6-9 years old, $4=9-12$ years old, and $5=12$ years old or older.

Finally, for the WA task, participants were asked to write down the first word that came to mind when they heard each word and were told that these words must be different from the original word. The participants followed the next instruction: "You will listen to a list of words. As soon as you hear each word, write down the first word that comes to your mind according to the word presented. The word you write down must be different from the original word." Two examples, in which filler stimuli and responses were used, were presented to clarify the task.

\section{RESULTS}

Different statistical procedures were applied to the data obtained from variables that used the 5-point numeric scales (IA, FAM, Comp., IV, and AoA) and to the data of the variables corresponding to nominal scales, such as NA and WA. For NA and WA, the percentage of the most frequent name (\%MFN) used, the word most associated, and a dispersion measure- the $H$-statistic (taken from Snodgrass \& Vanderwart, 1980)—were calculated. 
After that, we calculated all the descriptive ratings for all the variables $(M$, median, $S D$, minimum value, maximum value, range, 25 th percentile, 75 th percentile, interquartile range, asymmetry, and kurtosis) to analyze the behavior of the variables in terms of distribution and variability. For the calculation of the rating's incorrect answers, lost cases (i.e., no answers [blank spaces] and answers that included DKO, DKN, TOT, NI, and DO) were eliminated. Additionally, we calculated the Pearson correlation coefficient $(r)$ to determine the relation among the variables, as well as the correlation between this investigation and those performed with Spanish, English, and French samples. In order to analyze the errors, we calculated the percentage of wrong answers corresponding to the variables for each picture.

\section{Descriptive Analysis}

A summary of the rating data obtained from our samples of Spanish-speaking participants is presented in Appendix A of the supplemental material. This database contains each picture, with its most frequent name given in our population (first column), where the names are presented in alphabetical order with their corresponding numbers. The words in parentheses were added (e.g., banquito), to distinguish between those pictures (e.g., for numbers 33 and 34) that had obtained the same name (banco, for both) in the name-agreement task. We show its corresponding name in English (second column), the two name-agreement measures ( $H$ and \%MFN; third and fourth column, respectively), $M$, median, and $S D$ for each picture in the FAM, IA, Comp., IV, and AoA variables (in the subsequent columns). $H$ stands for WA, \%MFN, and the word(s) associated that had the greatest agreement (in the three last columns). Appendix B of the supplemental material presents free-association norms for Spanish names for the set of 400 figures taken from Cycowicz et al. (1997). For each stimulus, the norms include a complete numbered list of the participants' responses, ordered by proportion. For each independent response, total frequency (FREQUENCY) and the percentage and proportion of participants who gave that response (PERCENTAGEPROPORTION) are provided.
Next, summary statistics of each variable in the study are described (Table 1).

Although there are wide ranges in the ratings, there are no strong asymmetries; therefore, measures of central tendency (mean and median) are very close. The greatest asymmetries were found in variables $H / \mathrm{NA}$ and $\% \mathrm{MFN} / \mathrm{NA}$.

In the analysis of individual variables, it can be observed that $H$ values in NA show an important dispersion of names. A value of $H=0$ (perfect agreement) was obtained in only 102 pictures (25.5\% of the total pictures). Meanwhile, for \%MFN, an $M=80.97$ was obtained, with $S D=20.75$, indicating that many of the pictures were evaluated with the most frequent name relative to the total number of names given. However, the value found in the minimum range shows that some pictures were very difficult to name.

In relation to WA, $M=1.22$ and $S D=0.25$ in $H$ were obtained, which shows an elevated dispersion of alternative words associated with the names of the pictures. There are no pictures for which $H=0$ (unanimity in the answer), and only $25 \%$ of the pictures for which $H$ is below 1.04 . The low $\%$ MFN value is reflected by the fact that $75 \%$ of the pictures have values below $37 \%(Q 3=37)$. In addition, the minimum value found reveals that there are picture names that were very difficult to associate with a single word, and there was not a word found that could be associated with the name of a picture with an agreement of $100 \%$.

The data of the FAM variable indicate that the pictures were marked mainly as partially familiar, finding more dispersion among higher values.

Data obtained in IA suggest that the pictures evoke visual images similar to the pictures presented; $75 \%$ of the pictures had values higher than 3 , which indicates a medium to high concordance with the picture.

With respect to the Comp. variable, most pictures are considered as partially complex, with a data distribution approximate to normal.

IV shows that most pictures were evaluated as low variable, where $75 \%$ of the cases have a value lower than 2.9 , indicating that few different images are evoked by the name of a picture.

Table 1

Results of the Descriptive Ratings Obtained in Scales

\begin{tabular}{lrcccccccc}
\hline & $H / \mathrm{NA}$ & \%MFN/NA & H/AI & \%MFN/AI & FAM & IA & Comp. & IV & AoA \\
\hline Mean $(M)$ & 0.68 & 80.97 & 1.22 & 29.80 & 2.81 & 3.53 & 3.14 & 2.24 & 2.52 \\
Median & 0.57 & 89 & 1.22 & 26 & 2.66 & 3.68 & 3.14 & 2.00 & 2.43 \\
Standard deviation $(S D)$ & 0.61 & 20.75 & 0.25 & 15.29 & 1.17 & 1.14 & 0.81 & 1.09 & 0.79 \\
Asymmetry & 0.41 & -0.97 & 0.27 & 1.33 & 0.19 & -0.56 & 0.02 & 0.89 & 0.33 \\
Kurtosis & -1.17 & -0.18 & 1.17 & 1.87 & 1.05 & 0.26 & 0.57 & 0.86 & 0.05 \\
Range & 2.16 & 82 & 2.13 & 82 & 3.68 & 3.78 & 2.97 & 3.52 & 2.95 \\
Minimum value & 0.00 & 18 & 0.07 & 7 & 1.19 & 1.22 & 1.63 & 1.15 & 1.23 \\
Maximum value & 2.16 & 100 & 2.20 & 89 & 4.87 & 4.99 & 4.60 & 4.67 & 4.18 \\
25th percentile & 0.00 & 66 & 1.04 & 20 & 1.95 & 3 & 2.60 & 1.40 & 1.99 \\
75th percentile & 1.22 & 100 & 1.39 & 37 & 3.61 & 4 & 3.69 & 2.90 & 3.04 \\
Interquartile range & 1.22 & 34 & 0.35 & 17 & 1.66 & 2 & 1.09 & 1.50 & 1.06 \\
\hline
\end{tabular}

Note $-N=35$ for all; $H$, information statistic; NA, name agreement; \%MFN, percentage most frequent name; AI, association index; FAM, familiarity; IA, image agreement; Comp., visual complexity; IV, image variability; AoA, age of acquisition. 
Table 2

Bivariate Correlations Among Measures

\begin{tabular}{|c|c|c|c|c|c|c|c|c|c|}
\hline & $H / \mathrm{NA}$ & $\% \mathrm{MFN} / \mathrm{NA}$ & H/AI & $\% \mathrm{MFN} / \mathrm{AI}$ & IA & FAM & Comp. & IV & AoA \\
\hline$H / \mathrm{NA}$ & 1 & & & & & & & & \\
\hline$\% \mathrm{MFN} / \mathrm{NA}$ & $-.94^{* *}$ & 1 & & & & & & & \\
\hline$H / \mathrm{AI}$ & -.06 & .06 & 1 & & & & & & \\
\hline$\% \mathrm{MFN} / \mathrm{AI}$ & .00 & .02 & .03 & 1 & & & & & \\
\hline Image agreement (IA) & $-.31^{* *}$ & $.28^{* *}$ & -.01 & .04 & 1 & & & & \\
\hline Familiarity (FAM) & $-.27^{* *}$ & $.23^{* *}$ & $.17^{* *}$ & -.05 & .06 & 1 & & & \\
\hline Visual complexity (Comp.) & $.16^{* *}$ & $-.15^{* *}$ & -.08 & -.03 & $-.21^{* *}$ & $-.43^{* *}$ & 1 & & \\
\hline Image variability (IV) & -.15 & .12 & .06 & -.03 & $-.17^{* *}$ & $.60^{* *}$ & $-.24^{* *}$ & 1 & \\
\hline Age of acquisition (AoA) & $.37^{* *}$ & $-.34^{* *}$ & -.03 & .05 & .07 & $-.55^{* *}$ & $.25^{* *}$ & $-.57^{* *}$ & 1 \\
\hline
\end{tabular}

Note- $H$, information statistic; NA, name agreement; \%MFN, percentage most frequent name; AI, association index. ${ }^{* *} p<.01$.

In the AoA scale, an $M=2.52$ with an $S D=0.79$ was obtained, indicating that most of the picture names were learned at early ages (i.e., before the age of 9).

In relation to wrong answers, the total percentage was $13.82 \%$, which was due to some items having a relatively high rate of incorrect answers: calibrador ["calipers"], with $50 \%$ wrong answers, pretzel ["pretzel"] with $43 \%$, veleta ["weather vane"] with $38 \%$, azada ["hoe"] with $33 \%$, yunque ["anvil"] with $31 \%$, and pez globo ["blowfish"] with $29 \%$. The highest number of errors was in the NA task, with $7.4 \%$ for the total of the items. In the remaining variables, the rate was very low $(\mathrm{FAM}=2.5 \%$; $\mathrm{IA}=2.3 \%$; WA $=0.9 \% ; \mathrm{IV}=0.8 \%$ ).

\section{Correlation Among the Measures}

With the means obtained for each variable, a matrix of correlations for all variables under study was done for the calculation of the Pearson $r$ coefficient (Table 2).

In general, there are some high statistically significant correlations $(p<.01)$, although they are relatively small in magnitude. The exception is the relation found between $H / \mathrm{NA}$ and \%MFN/NA, which, as expected, had a strong negative correlation for being both complementary measures of NA and based on the same data. Additionally, moderate correlations were found between FAM and IV, FAM and AoA, FAM and Comp., and IV and AoA.

\section{Comparisons and Correlations \\ With Other Studies}

To compare our results with previous studies, we extracted 260 pictures in common between this study and the other investigations (with the exception of Alario \& Ferrand,
1999). We again calculated all the descriptive ratings for the 260 pictures in common, which are presented in Table 3.

In general, there are no notable discrepancies across the different studies. However, the rating obtained in the $H$ value by Sanfeliu and Fernández (1996) is lower than that obtained by most other studies, and the values of IA and FAM obtained by Manzano et al. (1997) and by Aveleyra et al. (1996), respectively, are higher than those presented in other investigations.

The comparison made with the study of Alario and Ferrand (1999) for the complete set of 400 pictures shows no considerable differences in the measures IA $(M=3.44)$, Comp. $(M=3.09)$, IV $(M=2.76)$, and AoA $(M=2.56)$. The NA variable $(M=0.36)$ obtained by Alario and Ferrand (1999) has an $H$ value nearer to 0, coinciding with a higher value of \%MFN $(M=84.63)$, whereas the FAM index $(M=2.72)$ had a lower value than did that of the present investigation.

Finally, the WA was compared with Fernández et al. (2004), which concentrated on the WA variable for the names of Snodgrass and Vanderwart's (1980) set of 260 pictures. This comparison was possible only for the associated pairs obtained, since, in the Spanish study, a dispersion measure of the given names $(H)$ was not calculated. Both studies coincided on the associated word in 39\% (same picture name and most associated word), with $28 \%$ of dissimilar associated pairs (same name of the picture, but they differ in the most associated word to the name) and 33\% of noncoincident pairs (they differ in the name given to the picture, and, therefore, the associated word varies too).

On the other hand, correlations among the overlapping variables and pictures obtained from the data of the pres-

Table 3

Descriptive Ratings of the 260 Pictures of the Snodgrass and Vanderwart (1980) Set

\begin{tabular}{|c|c|c|c|c|c|c|c|c|c|c|}
\hline \multirow[b]{2}{*}{ Variable } & \multicolumn{2}{|c|}{ Present Study } & \multicolumn{2}{|c|}{$\begin{array}{l}\text { Manzano } \\
\text { et al. (1997) }\end{array}$} & \multicolumn{2}{|c|}{$\begin{array}{l}\text { Aveleyra } \\
\text { et al. (1996) }\end{array}$} & \multicolumn{2}{|c|}{$\begin{array}{c}\text { Sanfeliu \& } \\
\text { Fernández } \\
\text { (1996) }\end{array}$} & \multicolumn{2}{|c|}{$\begin{array}{c}\text { Snodgrass \& } \\
\text { Vanderwart } \\
(1980)\end{array}$} \\
\hline & $M$ & $S D$ & $M$ & $S D$ & $M$ & $S D$ & $M$ & $S D$ & M & SD \\
\hline$H / \mathrm{NA}$ & 0.56 & 0.59 & 0.49 & 0.60 & - & - & 0.27 & 0.41 & 0.56 & 0.53 \\
\hline$\% \mathrm{MFN} / \mathrm{NA}$ & 84.80 & 19.10 & 85.11 & 20.64 & - & - & 82.3 & 21.68 & 86.59 & 14.34 \\
\hline Image agreement & 3.64 & 1.28 & 4.14 & 0.54 & 3.79 & 0.92 & 3.71 & 0.60 & 3.69 & 0.58 \\
\hline Familiarity & 3.03 & 1.56 & 3.47 & 0.88 & 4.20 & 0.99 & 3.12 & 1.11 & 3.29 & 0.96 \\
\hline Visual complexity & 2.58 & 1.24 & 2.29 & 0.66 & 2.70 & 0.94 & 2.67 & 0.93 & 2.96 & 0.89 \\
\hline Image variability & 2.24 & 1.26 & - & - & - & - & 2.61 & 0.59 & 2.61 & 0.59 \\
\hline
\end{tabular}

Note $-H$, information statistic; NA, name agreement; \%MFN, percentage most frequent name. 
Table 4

\begin{tabular}{|c|c|c|c|}
\hline & $\begin{array}{c}\text { French } \\
\text { (Alario \& } \\
\text { Ferrand, 1999) }\end{array}$ & $\begin{array}{c}\text { American } \\
\text { (Snodgrass \& } \\
\text { Vanderwart, 1980) }\end{array}$ & $\begin{array}{c}\text { Spanish } \\
\text { (Sanfeliu \& } \\
\text { Fernández, 1996) }\end{array}$ \\
\hline NA & .34 & .38 & .51 \\
\hline $\mathrm{MFN} / \mathrm{NA}$ & .37 & .30 & .54 \\
\hline miliarity & .87 & .81 & .72 \\
\hline nage agreement & .56 & .54 & .63 \\
\hline isual complexity & .88 & .92 & .51 \\
\hline nage variability & .72 & - & .54 \\
\hline ge of acquisition & .72 & - & - \\
\hline
\end{tabular}

Note-All values significant at $p<.01 ; H$, information statistic. NA, name agreement; \%MFN, percentage most frequent name.

ent study (mean values for each item) with those obtained previously (Alario \& Ferrand, 1999; Sanfeliu \& Fernández, 1996; Snodgrass \& Vanderwart, 1980) were calculated (see Table 4).

In general, it seems that high correlations found among pairs from different works would indicate the same rating pattern among the different populations in relation to each variable. FAM and Comp. had the strongest correlations, whereas the correlations of $H$ and \%MFN pairs had lower values. Moreover, the strongest correlations were found in the Comp. and FAM measures between this investigation and that of Snodgrass and Vanderwart (1980).

\section{DISCUSSION}

Normative data were obtained for a set of 400 pictorial stimuli, according to seven psycholinguistic variables: NA, IA, FAM, Comp., IV, AoA, and WA. These variables have significant theoretic importance for the study of $\operatorname{cog}$ nitive processes in language production. In addition, the norms obtained made it possible to observe the intrinsic relations among variables and to determine the most difficult tasks and the most problematic items in relation to the error percentage. It also made it possible to contrast the findings with those of other investigations, which contributed to a valid indicator with regard to the procedure and measurement of variables, and to discriminate which variables are most affected by language and culture.

In what follows, the general characteristics of the set of pictures are presented, together with the conclusions drawn from the correlations among measures and an analysis of the errors. We also present interpretations made from the comparisons and correlations of this investigation with previous studies.

In general, the complete set of pictures shows the following characteristics: An important number of pictures present a high rate of agreement with the name, although most were not named in a univocal way. Also, they show a high variability in relation to the words associated to each picture name, are considered partially familiar and partially complex, fit the mental image produced by their names, present a low degree of image variability, and, in most of the cases, are learned at early ages. However, due to the great number of pictures and the heterogeneous distribution of answers, items with different characteristics can be found. Thus, the set of pictures makes it possible to select many stimuli with specific particularities.

In relation to correlations among measures, low, moderate, and high associations were found, some of which were very significant. In general, this result allows us to suppose some interrelation between specific variables, which is expected, since there are variables that affect the same stages or phases of the object-naming process (such as the correlations found between NA and AoA, both of which would affect the retrieval phase of the phonological representation, or the one found between FAM and IV, which would influence the stage of access to the semantic representation). Nevertheless, the strong correlation found between $H / \mathrm{NA}$ and \%MFN/NA would be due to the fact that both are complementary measures of the same variable (NA) and to the fact that they function in an opposite way (while one increases, the other decreases). On the other hand, low correlations suggest that variables measuring different pictures' attributes are independent. In addition, Alario and Ferrand (1999) found a similar magnitude and direction of the correlations between the variables in a French sample.

The analysis of the wrong answers by variable showed the highest number of errors in NA. This may be due to the fact that the task of naming a picture is the most demanding one for the participants, since they have to access not only the structural representation but also the semantic and phonological structures (Humphreys, Riddoch, \& Quinlan, 1988). Additionally, the impact of errors on this variable was found in other studies (Manzano et al., 1997; Snodgrass \& Vanderwart, 1980).

The analysis of the pictures that obtained the most errors in each variable allowed us to discriminate a group of images that could be classified as problematic, owing to the high percentages of errors occurring in all the variables. The most problematic pictures were of calipers, a pretzel, a weather vane, a hoe, an anvil, and a blowfish, perhaps because the objects and/or concepts that they denote are not experienced daily for the participants in the present sample. Hence, these pictures should be discriminated from the remaining set when they are used as stimuli. 
The comparisons and correlations made between this study and the normative data obtained with Spanish-, English-, and French-speaking participants allowed us to evaluate the validity and reliability of the means obtained for each measure. In general, the same result patterns and high correlations were found, which would indicate the validity of our measures (as regards the testing procedure) and the reliability of the data obtained (congruency between data). At the same time, we hypothesize that the differences across studies are due to influences from the cultural and linguistic context. Some variables seem to be more sensitive to the context than are others.

The NA variable proved to be the most dependent on language and/or culture, since it obtained the most diverse values and the lowest correlations between this study and previous studies in Spanish, French, and English. Similar results have been reported by all the studies in which formally equivalent comparisons have been performed (e.g., Alario \& Ferrand, 1999; Dell'Acqua et al., 2000; Sanfeliu \& Fernández, 1996). This pattern of results was expected, on the assumption that NA values depend on language more than the other measures do (Dell'Acqua et al., 2000). The reason for this may be that, when selecting the word that will name an object, an exclusive use of the language is performed, which influences the idiosyncratic way of naming objects. It is here that NA plays an important role as a psycholinguistic predictor variable in this stage of the process.

The WA is another variable that seems to be highly influenced by dialect and by cultural context. Comparison between this study and the one by Fernández et al. (2004) allowed us to observe that, although these studies studied the same language, considerable differences were found among the associated words. These differences are very common among people who share a language but live in different communities, such as those in French between France and Canada (Alario \& Ferrand, 1999, and Sirois, Kremin, \& Cohen, 2006, respectively) and in English between England and the United States (Barry et al., 1997, and Snodgrass \& Vanderwart, 1980, respectively). Furthermore, as shown by Bates et al. (2003), the differences in these types of studies comparing languages can be related to a large variety of reasons, including cultural variations in the frequency of or accessibility to a particular concept.

The FAM variable produced the highest correlations between this study and the English, French, and Spanish studies. This could be explained by the nature of the items that appeared in each of the sets of drawings used in each work, since the material used in the four investigations includes the Snodgrass and Vanderwart (1980) set, in which the pictures with high values of typicality were chosen (Battig \& Montague, 1969). The pattern shared by the different populations in the study could be due to the fact that a great number of pictures of the set are very typical objects in any culture, time, and language (e.g., spoon, fork, chair, table; Székely et al., 2003).

For the Comp. variable, it is important to notice the high values found in the correlations between this work and the
English and French samples specifically, which could be due to the characteristics of the task, in which participants evaluated the object on the basis of the quantity of details and lines presented. Thus, no important variation would be expected in the answer pattern of the participants when they belong to different cultures, because the objectivity of the task requires evaluating the object and not the word used to name it.

Finally, it is necessary to point out that picture naming is a very frequent task used in psychology and neuropsychology for experiments and diagnoses; therefore, the availability of these norms in the field will allow researchers to rigorously select the material to use in this kind of test.

\section{AUTHOR NOTE}

Address correspondence to L. Manoiloff, Laboratorio de Psicología Cognitiva, Facultad de Psicología, Universidad Nacional de Córdoba, Enrique Barros y Enfermera Gordillo, CP 5000, Córdoba, Argentina (e-mail: 1manoiloff@psyche.unc.edu.ar).

\section{REFERENCES}

Alario, F.-X., \& FERrand, L. (1999). A set of 400 pictures standardized for French: Norms for name agreement, image agreement, familiarity, visual complexity, image variability, and age of acquisition. Behavior Research Methods, Instruments, \& Computers, 31, 531-552.

Alario, F.-X., Ferrand, L., Laganaro, M., New, B., Frauenfelder, U. H., \& SEgui, J. (2004). Predictors of picture naming speed. Behavior Research Methods, Instruments, \& Computers, 36, 140-155.

Aveleyra, E., Gómez, C., Ostrosky, F., \& Rigalt, C. (1996). Adaptación de los estímulos no verbales de Snodgrass y Vanderwart en población de hispanohablante: Criterios para la denominación, concordancia de la imagen, familiaridad y complejidad visual. Revista Mexicana de Psicología, 13, 5-19.

Barbón, A., \& Cuetos, F. (2006). Efectos de la edad de adquisición en tareas de categorización semántica. Psicológica, 27, 207-223.

Barry, C., Morrison, C. M., \& Ellis, A. W. (1997). Naming the Snodgrass and Vanderwart pictures: Effects of age of acquisition, frequency, and name agreement. Quarterly Journal of Experimental Psychology, 50A, 560-585. doi:10.1080/027249897392026

Bates, E., D’Amico, S., Jacobsen, T., Székely, A., Andonova, E., DeVESCOVI, A., ET AL. (2003). Timed picture naming in seven languages. Psychonomic Bulletin \& Review, 10, 344-380.

Battig, W. F., \& Montague, W. E. (1969). Category norms for verbal items in 56 categories: A replication and extension of the Connecticut category norms. Journal of Experimental Psychology, 80, 1-46. doi:10.1037/h0027577

Bonin, P., Boyer, B., Méot, A., Fayol, M., \& Droit, S. (2004). Psycholinguistic norms for action photographs in French and their relationships with spoken and written latencies. Behavior Research Methods, Instruments, \& Computers, 36, 127-139.

Cuetos, F., Ellis, A. W., \& Alvarez, B. (1999). Naming times for the Snodgrass and Vanderwart pictures in Spanish. Behavior Research Methods, Instruments, \& Computers, 31, 650-658.

Cycowicz, Y. M., Friedman, D., Rothstein, M., \& Snodgrass, J. G. (1997). Picture naming by young children: Norms for name agreement, familiarity, and visual complexity. Journal of Experimental Child Psychology, 65, 171-237. doi:10.1006/jecp.1996.2356

Dell'ACQuA, R., LotTo, L., \& Job, R. (2000). Naming times and standardized norms for the Italian PD/DPSS set of 266 pictures: Direct comparisons with American, English, French, and Spanish published databases. Behavior Research Methods, Instruments, \& Computers, 32, 588-615.

Dent, K., Johnston, R. A., \& Humphreys, G. W. (2008). Age of acquisition and word frequency effects in picture naming: A dual-task investigation. Journal of Experimental Psychology: Learning, Memory, \& Cognition, 34, 282-301. doi:10.1037/0278-7393.34.2.282

Ellis, A. W., \& Morrison, C. M. (1998). Real age-of-acquisition ef- 
fects in lexical retrieval. Journal of Experimental Psychology: Learning, Memory, \& Cognition, 24, 515-523. doi:10.1037/0278-7393.24 .2 .515

Fernández, A., Diez, E., Alonso, M. A., \& Beato, M. S. (2004). Free-association norms for the Spanish names for the Snodgrass and Vanderwart pictures. Behavior Research Methods, Instruments, \& Computers, 36, 577-583.

Hirsh, K. W., \& FunNell, E. (1995). Those old, familiar things: Age of acquisition, familiarity, and lexical access in progressive aphasia. Journal of Neurolinguistics, 9, 23-32. doi:10.1016/0911-6044(95)00003-8

Humphreys, G. W., Riddoch, M. J., \& Quinlan, P. T. (1988). Cascade processes in picture identification. Cognitive Neuropsychology, 5, 67104. doi: $10.1080 / 02643298808252927$

Iyer, G. K., Saccuman, C. M., Bates, E. A., \& Wulfeck, B. B. (2001). A study of age-of-acquisition (AoA) ratings in adults. Center for Research in Language Newsletter, 13, 3-32.

Manzano, M., Piñeiro, A., \& Reigosa, V. (1997). Estudio de las características de un conjunto de 260 figuras en sujetos adultos de habla hispana. Cognitiva, 9, 29-64. doi:10.1174/02143559760422370

Morrison, C. M., Chappell, T. D., \& Ellis, A. W. (1997). Age of acquisition norms for a large set of object names and their relation to adult estimates and other variables. Quarterly Journal of Experimental Psychology, 50A, 528-559. doi:10.1080/027249897392017

Morrison, C. M., \& ElLis, A. W. (1995). Roles of word frequency and age of acquisition in word naming and lexical decision. Journal of Experimental Psychology: Learning, Memory, \& Cognition, 21, 116133. doi:10.1037/0278-7393.21.1.116

Morrison, C. M., Ellis, A. W., \& Quinlan, P. T. (1992). Age of acquisition, not word frequency, affects object naming, not object recognition. Memory \& Cognition, 20, 705-714.

Pérez, M. A., \& Navalón, C. (2003). Normas españolas de 290 nuevos dibujos: Acuerdo en la denominación, concordancia de la imagen, familiaridad, complejidad visual, y variabilidad de la imagen. Psicológica, 24, 215-241.

Sanfeliu, M. C., \& Fernández, A. (1996). A set of 254 Snodgrass-
Vanderwart pictures standardized for Spanish: Norms for name agreement, image agreement, familiarity, and visual complexity. Behavior Research Methods, Instruments, \& Computers, 28, 537-555.

Sirois, M., Kremin, H., \& Cohen, H. (2006). Picture-naming norms for Canadian French: Name agreement, familiarity, visual complexity, and age of acquisition. Behavior Research Methods, 38, 300-306.

SNOdgrass, J. G., \& VANDERWART, M. (1980). A standardized set of 260 pictures: Norms for name agreement, image agreement, familiarity, and visual complexity. Journal of Experimental Psychology: Human Learning \& Memory, 6, 174-215. doi:10.1037/0278-7393.6.2.174

Snodgrass, J. G., \& Yuditsky, T. (1996). Naming times for the Snodgrass and Vanderwart pictures. Behavior Research Methods, Instruments, \& Computers, 28, 516-536.

Székely, A., D’Amico, S., Devescovi, A., Federmeier, K., HerRON, D., IYER, G., ET AL. (2003). Timed picture naming: Extended norms and validation against previous studies. Behavior Research Methods, Instruments, \& Computers, 35, 621-633.

Vitkovitch, M., \& Tyrrell, L. (1995). Sources of disagreement in object naming. Quarterly Journal of Experimental Psychology, 48A $822-848$

\section{NOTE}

1. The instructions were given in Spanish.

\section{SUPPLEMENTAL MATERIALS}

The Spanish picture norms discussed in this article, in Excel and .pdf format, may be downloaded from www.psyche.unc.edu.ar/investigacion/ normas_pictures.php, or as supplemental materials from http://brm .psychonomic-journals.org/content/supplemental.

(Manuscript received May 12, 2009; revision accepted for publication November 14, 2009.) 